\title{
A IMPORTÂNCIA DA EQUIPE INTERDISCIPLINAR NO DIAGNÓSTICO DE DISLEXIA DO DESENVOLVIMENTO
}

\section{Relato de caso}

\author{
Magda S. Vanzo Pestun', Sylvia Ciasca², Vanda Maria Gimenes Gonçalves ${ }^{3}$
}

\begin{abstract}
RESUMO - Descrevemos o trabalho de uma equipe interdisciplinar da FCM/UNICAMP na avaliação de um menino de 9 anos e 3 meses aluno de escola pública de Campinas, com dislexia do desenvolvimento. 0 procedimento constou de 4 fases: 1) anamnese com a mãe; 2) avaliação neuropsicológica; 3) avaliação específica de leitura e escrita; 4) exames complementares. Os resultados das várias avaliações revelaram que o menino apresenta inteligência normal, ausência de déficit auditivo ou visual mas dificuldades em provas específicas de leitura, em memória de curto prazo (especialmente em sequências auditivas) e em consciência fonológica, além de lentidão, falta de concentração, sinais neurológicos menores e hipoperfusão da porção mesial do lobo temporal. Esses dados sugeriram que a criança apresenta dislexia do desenvolvimento do tipo misto, necessitando de intervenção psicopedagógica.
\end{abstract}

PALAVRAS-CHAVE: dislexia do desenvolvimento, equipe interdisciplinar, diagnóstico.

The importance of interdisciplinary staff in the diagnosis of developmental dyslexia: case report

\begin{abstract}
We describe the work of the interdisciplinary staff of FCM/UNICAMP for the diagnosis of developmental dyslexia, evaluating a 9 years old boy from the second year of a first grade public school. The procedure consisted of four stages: 1) Interview with the mother (anamnesis); 2) neuropsychological evaluation; 3) specific evaluation for reading and writing skills; 4) complementary exams. The results revealed that the child presented normal intelligence, normal auditory and visual function but difficulties in reading specific test, in auditory short-term memory (specially in auditory sequences), and in phonological conscience, as well as slowness, lack of concentration, slight neurological signs and hypoperfusion of the mesial portion of the temporal lobe. These data suggest that the child has developmental dyslexia of mixed type, requiring psychopedagogic follow-up.
\end{abstract}

KEY WORDS: developmental dyslexia, interdisciplinary staff, diagnosis.

Na ciência do século XXI é essencial a troca de conhecimentos entre as várias disciplinas. No caso dos distúrbios específicos do aprendizado, e em especial na dislexia do desenvolvimento, a troca de informações entreas áreas médica, neuropsicológica e pedagógica é fundamental para promover a interdisciplinariedade e, consequentemente, ampliar nosso conhecimento acerca dessas disfunções. O órgão responsável pela aprendizagem é o cérebro ${ }^{1}$. A aprendizagem, capacidade de processar, armazenar e usar a informação é, portanto, uma função cerebral. No caso da leitura, processo altamente complexo, estão envolvidos tanto aspectos neurológicos como sensoriais, psicológicos, sócio-culturais, sócioeconômicos e educacionais, dentre outros. Portan- to, um único profissional não basta para analisar esses vários aspectos e fornecer diagnóstico preciso e seguro. Dislexia do desenvolvimento é disfunção do sistema nervoso central, frequentemente de origem constitucional, caracterizada pela dificuldade na aquisição ou no uso da leitura e/ou escrita, que acomete crianças com inteligência normal, sem défices sensoriais, com instrução supostamente adequada e na ausência de problemas físicos ou emocionais significativos ${ }^{2-5}$.

Boder $(1973)^{6}$ distinguiu três tipos de disléxicos (disfonéticos, diseidéticos emistos) baseando-se nos tipos de erros produzidos nas diversas condições de leitura (palavras familiares ou não familiares, longas ou curtas, de alta ou baixa frequência). Os disléxicos

Departamento de Neurologia, Faculdade de Ciências Médicas (FCM), Universidade de Campinas (UNICAMP), Campinas SP, Brasil: ${ }^{1}$ Doutoranda em Neurologia Infantil; ${ }^{2}$ Professora Assistente Doutora; ${ }^{3}$ Professora Doutora.

Recebido 17 Julho 2001, recebido na forma final 26 Outubro 2001. Aceito 9 Novembro 2001. 
disfonéticos lêem bem as palavras que eles conhecem, ou seja, que memorizam visualmente, mas não lêem nem escrevem palavras que encontram pela primeira vez. Eles as advinham, a partir do contexto e de indicações como a letra inicial ou a extensão da palavra, e cometem muitos erros de substituição semântica. Os disléxicos diseidéticos caracterizamse por apresentarem uma leitura lenta, trabalhosa, mas correta, baseada na decodificação fonética. Lêem tanto palavras familiares quanto não familiares, mas apresentam dificuldade em palavras irregulares. Os disléxicos mistos reúnem as dificuldades desses dois tipos e frequentemente apresentam confusões espaciais. Pela própria conceituação, há necessidade de que um grupo de profissionais proceda à investigação eà análise dos déficits funcionais, trace o perfil de desempenho da criança, formule hipóteses explicativas e especifique os objetivos terapêuticos. O psicólogo conduzirá a avaliação emocional, perceptual e intelectual. O pedagogo fará a avaliação acadêmica. A fonoaudióloga poderá conduzir a avaliação audiométrica cujo objetivo é descartar possível défice auditivo. O médico oftalmologista realizará o exame de acuidade visual, cujo objetivo é excluir défice visual. O médico neurologista irá realizar o exame neurológico tradicional (ENT) e o evolutivo (ENE), afastando o comprometimento neurológico. O ENT utiliza técnica semiológica voltada para detectar síndromes neurológicas ou anormalidades neurológicas maiores em lesões específicas de núcleos, tratos ou nervos cranianos. Permite também observar alguns sinais neurológicos menores (soft signs) ${ }^{7}$. Neste conceito estão incluídos fenômenos como a astereognosia, disdiadococinesia, movimentos em espelho e extinção. O ENE tem por objetivo aferir a maturidade neurológica através da avaliação da lateralidade, equilíbrio estático e dinâmico, coordenação apendicular, coordenação troncomembros, sensibilidade/gnosias, persistência motora e linguagem ${ }^{8}$. O neurorradiologista poderá conduzir a avaliação por imagem (com ressonância magnética - RM, tomografia computadorizada por emissão de fóton único - SPECT, tomografia por emissão de pósitrons- PET). Osdados fornecidos por estesprofissionais poderão contribuir com novas informações ou descartar possíveis lesões. Os resultados da avaliação de cada profissional deverão ser analisadose discutidos por todos e deverá ser proposto um diagnóstico diferencial, especificando as integridadese dificuldades observadas na criança.

Este trabalho tem como objetivo descrever um caso ilustrativo quanto ao trabalho interdisciplinar no diagnóstico de dislexia do desenvolvimento rea- lizado no Ambulatório de Neuro-Dificuldades de Aprendizagem, Disciplina de Neurologia Infantil, FCM- UNICAMP.

\section{MÉTODO}

Caso

Menino de 9 anos e 3 meses, destro, aluno da segunda série do primeiro grau de escola pública da região de Campinas/SP, de nível sócio-cultural e econômico médiobaixo, foi encaminhado pela escola ao Hospital de Clínicas (HC) da UNICAMP, para avaliação e diagnóstico, com queixa de fracasso na aquisição da leitura e escrita. Há 3 anos aproximadamente, uma equipe pedagógica tentava vários recursos sem resultados satisfatórios. Segundo a professora, a criança não presta atenção às explicações quando a atividade envolve raciocínio, não se concentra na execução dastarefas, não conclui as atividades no tempo previsto, não se expressa oralmente de forma clara, apresenta dificuldade em reproduzir fatos ou acontecimentos e não sabe transmitir recados. Não lê palavras complexas e a escrita apresenta muitos erros.

\section{Avaliação}

A avaliação foi realizada no Ambulatório de NeuroDificuldades de Aprendizagem, FCM - UNICAMP. Para avaliação utilizaram-se: a) testes formais: escala de inteligência Wechesler para crianças (WISC) ${ }^{9}$; teste guestáltico visomotor (Bender/Clawson) ${ }^{10}$; bateria Luria-Nebraska - avaliação neuropsicológica para crianças ${ }^{11}$; teste de audibilização ${ }^{12}$; tarefa de segmentação fonêmica ${ }^{13}$; listas de palavras para leitura oral, repetição e tomada de ditado ${ }^{14}$; texto para leitura oral e interpretação ${ }^{15}$; b) materiais de uso acadêmico (como lápis, papel sulfite, borracha); folhas de respostas; cronômetro e c) exames complementares: audiométrico; oftalmológico; neurológico tradicional (ENT) $)^{16}$ e evolutivo - versão abreviada (ENEVA) ${ }^{17}$; e cintilográfico (SPECT). As imagens tomográficas do cérebro foram adquiridas e reconstruídas nos planos transversal, coronal, temporal e sagital, após a injeção venosa de HMPAO99mTc.

O procedimento de diagnóstico constou de 4 etapas. $\mathrm{Na}$ etapa 1 foram realizadas a anamnese com a mãe da criança, com o objetivo de obter o máximo de informações possíveis a respeito das condições de gravidez, nascimento e desenvolvimento neuropsicomotor. Foi solicitada a assinatura do Termo de Consentimento Livre e Esclarecido para participação na pesquisa. Na etapa 2 foram realizadas as avaliações do potencial intelectual ${ }^{9}$, da maturação percepto visuo-motora ${ }^{10}$, da percepção auditiva ${ }^{12}$ e neuropsicológica (bateria Lúria-Nebraska) ${ }^{11}$. Na etapa 3 foi realizada a avaliação específica de leitura (leitura oral de 192 palavras, com e sem sentido, regulares, irregulares e regra, de alta e baixa frequência); leitura oral de texto com interpretação; tomada de ditado e repetição das mesmas 192 palavras empregadas na leitura; e tarefa de segmentação fonêmica, que envolvia delação e adição de fonemas, nos segmentos iniciais, mediais e finais, nas categorias de 
palavras reais para reais, de reais para pseudo palavras, de pseudo para reais e de pseudo para pseudo). Na etapa 4 foram realizadas as avaliações interdisciplinares (exames neurológico, audiométrico, oftalmológico e de imagem).

\section{RESULTADOS}

Para melhor compreensão, os resultados serão descritos seguindo a ordem do procedimento, ou seja, etapa por etapa.

Na anamnese, não foram relatadas intercorrências durante a gravidez; o parto foi longo e difícil, necessitando o uso de fórceps (a mãe não soube precisar se o fórceps foi de alívio ou alto). O desenvolvimento neuropsicomotor foi normal. A criança emitiu as primeiras palavras com significado com 10 meses e andou sem apoio com 11 meses de idade. Aos 2 anos apresentou convulsão, sendo medicado; a mãe não soube indicar o medicamento prescrito na ocasião. Aos 3 anos, apresentou uma segunda e última convulsão, sendo então submetido a radiografia de crânio, com resultado "normal". A partir de então, não mais necessitou de acompanhamento médico nem apresentou qualquer outra intercorrência. A mãe não soube especificar o tipo de convulsão.

$\mathrm{Na}$ avaliação intelectual, o menino apresentou desempenho médio para sua idade cronológica e nível sócio-cultural. Demonstrou escores reduzidos em aritmética, codificação, informação e dígitos (padrão ACID). O padrão ACID tem sido considerado como preditivo de desempenho fraco em leitura, ortografia e aritmética ${ }^{1,2}$. Na bateria Luria-Nebraska, demonstrou boa coordenação motora global e leve prejuízo em coordenação motora fina. Imitou e reproduziu gestos simbólicos e estruturas rítmicas. Discriminou objetos pelo tato, nomeou partes do corpo, reconheceu cores, objetos familiares e formas geométricas. Demonstrou boa gnosia e praxia visual. Quanto a lateralidade, reconheceu direita e esquerda tanto em si como no outro. A linguagem receptiva e expressiva se mostraram adequadas. A memória imediata (auditiva e visual) está limitada a três elementos. O teste Bender indicou atraso maturacional (idade mental dois anos abaixo da idade cronológica) e desenvolvimento perceptual irregular, sugerindo comprometimento funcional. No teste de audibilização, apresentou boa discriminação fonemática ( $92 \%$ de acerto), razoável conceituação (70\% de acerto) e bom vocabulário visual (83\% de acerto). Apresentou também nesta prova dificuldade em memória e síntese auditiva (não retém sequência de palavras e não consegue fazer síntese oral).

Quanto à leitura, leu melhor palavras de alta (71\% de acerto) do que de baixa frequência ( $56 \%$ de acer- to), regulares (81\% de acerto) do que irregulares ( $50 \%$ de acerto) e regras ( $59 \%$ de acerto). Apresentou dificuldade em leitura de pseudopalavras (palavras sem sentido - $46 \%$ de acerto). Apresentou muitos erros por substituição, tanto visuais quanto sonoros. Em leitura de texto, apresentou leitura silabada, sem respeito a sinais de pontuação e vários erros por substituição de fonemas tendo, no entanto, boa compreensão geral do mesmo. Levou $2 \mathrm{mi}-$ nutos e 50 segundos para realizar a tarefa. Seu desempenho em leitura de texto foi compatível como esperado para a primeira série ${ }^{18}$.

Em tomada de ditado, apresentou baixo desempenho sem diferença significativa entre palavras de alta frequência ( $29 \%$ deacerto) e de baixa frequência (31\% de acerto). Não houve também diferença de desempenho entre palavras familiares (30\% deacerto) e pseudopalavras ( $31 \%$ de acerto). As palavras regulares foram escritas de forma mais correta ( $56 \%$ de acerto) do que as palavras regra ( $19 \%$ de acerto) e palavras irregulares (15,5\% de acerto). Apresentou muitas trocas fonológicas em tomada de ditado (por exemplo: nosbra em vez de nosdra, jupeta em vez de chupeta, golegas em vez de colegas, vazendo em vez de fazendo). Realizou escrita espontânea de palavras simples e cópia com transcrição de letra de imprensa para cursiva.

Apresentou bom desempenho em repetição de palavras, tanto familiares (99\% de acerto) quanto pseudopalavras ( $97 \%$ de acerto).

Na tarefa de segmentação fonêmica, não apresentou consciência fonológica e teve dificuldade principalmente em deleção e adição de segmentos no início da palavra (obteve somente $28 \%$ de acerto). Manipulou melhor segmentos finais ( $62,5 \%$ de acerto) seguidos dos mediais (37\% de acerto).

Os exames oftalmológico e audiométrico apresentaram resultados normais, não tendo sido detectado défice sensorial. No exame neurológico, o ENT detectou microcefalia (perímetro craniano medindo $49,5 \mathrm{~cm}$ ) e exoforia no olho esquerdo. Ao ENEVA, foi observada disdiadococinesia bilateral; não reconheceu mão direita/esquerda. Seu perfil neurológico de desenvolvimento foi compatível com 6 anos em coordenação apendicular e com 5 anos em sensibilidade/gnosias. Realizou provas máximasnosdemais setores. No exame de imagem (SPECT), apresentou hipoperfusão da porção mesial do lobo temporal .

\section{DISCUSSÃO}

O ato de ler envolve a discriminação visual de símbolos gráficos através de um processo de deco- 
dicação. Esse processo exige atenção seletiva. Em seguida, há necessidade de selecionar e identificar os equivalentes auditivos (fonemas) através de um processo de análise e transdução, síntese e comparação, a fim de obter significado ${ }^{1}$. Portanto, a leitura envolve tanto a integridade do processamento visual quanto fonológico. Os resultados acima descritos sugerem que o menino estudado, apresenta prejuízos tanto no processamento fonológico quanto no processamento visual, comprometendo a leitura oral de palavras familiares (principalmente palavras irregulares) e pseudopalavras, leitura oral de texto e escrita. A dificuldade em decodificar palavras não familiares ou pseudopalavras (verificadas na leitura oral) e a insensibilidade fonológica ou a dificuldade em segmentar segmentos fonêmicos de palavras faladas (verificada na tarefa de segmentação fonêmica) são caracterizadas como desordens fonológicas, muito frequente em disléxicos ${ }^{19-23}$.

As dificuldades atencionais e sequenciais observadas nos testes de audibilização e WISC, principalmente nas provas de memória de dígitos e codificação que requerem manipulação mental de símbolos e alerta de atenção, são sinais característicos de distúrbio específico de linguagem, principalmente de leitura ${ }^{24}$. Como mencionado acima, a atenção seletiva e sustentada é fundamental no ato de ler. A dificuldade na retenção e recuperação das informações verificada nos testes de audibilização (memória para dígitos, frases e historietas), na Bateria LuriaNebraska (memória visual e auditiva) e WISC (números), a lentidão na execução das tarefas, observada em várias provas (leitura oral de texto, escrita de palavras) e distratibilidade são sinais indicativos de dislexia ${ }^{25,26}$. A ausência de défice sensorial (auditivo ou visual), constatado pelo oftalmologista e pela fonoaudióloga, foi fundamental para excluir prejuízo nas áreas receptivas, incompatível com dislexia.

O exame neurológico permitiu verificar ausência de comprometimento de grandes vias que também seria incompatível com distúrbio específico de leitura. É importante salientar a presença de sinais neurológicos menores (disdiadococinesia, agnosia direita-esquerda, exoforia à esquerda). Também é interessante observar que o domínio do conceito de direita e esquerda não está ainda estabelecido visto que, em algumas situações, a criança responde apropriadamente (Bateria Luria-Nebraska) e em outras, erroneamente (ENT), sugerindo que as respostas foram dadas ao acaso. Segundo a literatura ${ }^{1}$, o reconhecimento direita/esquerda refere-se ao poder de discriminação e verbalização que a criança tem do seu corpo como um universo espacial interiorizado e deve estar estabelecida entre 5 e 6 anos de idade (localização bilateral) e entre 7 e 9 anos (localização contralateral - cruzamento da linha média do corpo e reversível - localização no outro).

A microcefalia não interferiu no desempenho intelectual. Esses achados neurológicos foram interpretados como sinais menores isolados, não sendo identificada qualquer síndrome.

O SPECT, ao revelar hipoperfusão da porção mesial do lobo temporal, ou seja, redução do fluxo sanguíneo cerebral regional, sugere baixa utilização de glicose e um baixo metabolismo oxidativo. Devido a essa correlação, a medida do fluxo sanguíneo cerebral éuma forma de verificação do funcionamento cerebral in vivo ${ }^{27}$. Apesar de serem necessários mais estudos com neuroimagem funcional, os resultados obtidos sugerem alteração no funcionamento de uma região altamente envolvida no ato de ler. A porção mesial do lobo temporal realiza o processamento, integração e armazenamento da informação auditiva, bem como o reconhecimento da palavra escrita. Foi realizado estudo ${ }^{28}$ com PET para investigar mudanças no fluxo sanguíneo cerebral regional (rCBF) em sujeitos neurologicamente normais, durante leitura e repetição de palavras. A leitura de palavras causou aumento significativo no fluxo sanguíneo na parte posterior do giro temporal médio esquerdo e a repetição de palavras resultou na ativação bilateral do giro temporal superior. Esses resultados são consistentes com a localização de um léxico para reconhecimento de palavras escritas na parte posterior do giro temporal médio esquerdo e um léxico para reconhecimento de palavra falada na parte média do giro temporal superior e médio esquerdo. O processamento dos sons da fala éfortemente dependente do funcionamento intacto das partes posterior e superior do lobo temporal esquerdo. Pesquisas ${ }^{29,30}$ têm verificado pequeno grau de assimetria entre áreas e volumes dos lobos temporal em disléxicos quando comparados com sujeitos controle. Na última década, estudos neuropsicológicos sugerem que o substrato neurológico da leitura parece envolver mais do que o giro superior do lobo temporal. Um estudo ${ }^{5}$ empregando MRI em grupos de disléxicos $(n=17)$, leitores atrasados $(n=6)$ esujeitos controle $(n=12)$, encontrou diferenças mais pronunciadas nas medidas das áreas de cruzamento regional do córtex temporal com a substância branca subcortical, em particular a lateral da insula. Enquanto muitos dos leitores normais e atrasados (72\%) tinham assimetria esquerda, a maioria dos dis- 
léxicos (83\%) tinham assimetria direita ou medidas simétricas. Segundo esses autores, a ausência de assimetria esquerda pode estar associada com habilidades rebaixadas em análise fonêmica mais do que em discriminação fonológica. É interessante ressaltar que a criança deste estudo também apresentou dificuldades em análise fonêmica (memória de curto prazo, sequenciação e síntese auditiva) e bom desempenho em discriminação fonológica.

Em conclusão, alterações no processamento visual e auditivo, no funcionamento do lobo temporal, dificuldade na armazenagem e recuperação das informações, distratibilidade, ausência de déficessensoriais (auditivos e visuais) e presença de sinais neurológicos menores sugerem que o menino estudado apresenta dislexia do desenvolvimento do tipo misto (com prejuízos tanto fonológico quanto visual). A análise das avaliações realizadas por psicóloga, fonoaudióloga, oftalmologista, neurologista e radiologista permitiram elaborar o diagnóstico de dislexia e encaminhar a criança para acompanhamento psicopedagógico. Através do estudo deste caso queremos enfatizar que, na avaliação do paciente disléxico em língua portuguesa, os testes informais preconizados por Golbert ${ }^{12}$, Pinheiro ${ }^{14}$, Capovilla e col. ${ }^{13}$, associados à avaliação neurológica, sensorial e neuropsicológica formal, são essenciais no estabelecimento do diagnóstico e da conduta terapêutica. Além disso, a realização de exames de neuroimagem, sobretudo de caráter funcional, contribui para melhor compreensão dos mecanismos fisiopatológicos dos distúrbios do desenvolvimento.

Agradecimentos - Aos Serviços de Neuroimagem, Oftalmologia e Otorrinolaringologia/ FCM-UNICAMP, pela colaboração na avaliação do sujeito.

\section{REFERÊNCIAS}

1. Fonseca V. Introdução às dificuldades de aprendizagem. Porto Alegre: Artes Médicas, 1995.

2. Boder E. Developmental dyslexia: a diagnostic approach based on three atypical reading-spelling patterns. Dev Med Child Neurol 1973;21:504-514.

3. Garcia N. Dificultades de aprendizage: lenguaje, lecto-escritura y matemáticas. Madrid: Narcea, 1995.

4. Ellis AW. Leitura, escrita e dislexia: uma análise cognitiva. Porto Alegre: Artes Médicas, 1995.

5. Heilman KM, Voeller K, Alexander AW. Developmental dyslexia: a motor-articulatory feedback hipothesis. Ann Neurol 1996;39:407-412.
6. Dalby MA, Elbro C, Stodkilde J. Temporal lobe asymmetry and dyslexia: an in vivo study using MRI. Brain Language 1998;62:51-59.

7. Guardiola A, Ferreira LTC, Rotta NT. Associação entre desempenho das funções corticais e alfabetização em uma amostra de escolares de primeira série de Porto Alegre. Arq Neuropsiquiatr 1998;56:281-288.

8. Gonçalves VMG, Tonelotto JMF , Ravanini SG. Semiologia neurológica numa população de escolares da primeira série do ensino fundamental. Arq Neuropsiquiatr 2000;58:112-118.

9. Wechsler D. Mannual for the Wechsler Intelligence Scale for Children. New York: Psychological Corporation, 1974.

10. Clawson A. Bender Infantil: manual de diagnóstico clínico. Jurema Alcides Cunha. Porto Alegre: Artes Médicas, 1980.

11. Golden CY. The Nebraska Neuropsychological Children's Battery. In Reynolds CR, Fletcher-Jansen E (eds). Handbook of clinical child neuropsychology. New York: Plenum Press 1989;11:193-204.

12. Golbert CS. A evolução psicolinguística e suas implicações na alfabetização. Porto Alegre: Artes Médicas, 1988.

13. Capovilla FC, Colorni EMR, Nico AM, Capovilla AS. Leitura em voz alta, tomada de ditado, manipulação fonêmica, e relações entre elas: efeito de características de palavras (frequência, regularidade, lexicalidade) e de nível de escolaridade. Anais do II Congresso Brasileiro de Neuropsicologia. São Paulo, 1995.

14. Pinheiro AMV. Leitura e escrita: uma abordagem cognitiva. Campinas: PsyII, 1994.

15. Nahum EP. Meu caderno de redação. São Paulo: Scipione, 1991.

16. Lefèvre AB. O exame neurológico da criança. In Tolosa APM, Canelas HM (eds). Propedêutica neurológica. São Paulo: Sarvier, 1972.

17. Bachiega MCM. Exame neurológico evolutivo da criança normal de 3 a 7 anos de idade: contribuição para a validação da fidedignidade das provas. Dissertação de Mestrado. Faculdade de Medicina da Universidade de São Paulo. São Paulo, 1979.

18. Pestun MSV, Ciasca, S. Estudo piloto do nível de leitura em crianças de duas cidades brasileiras:comparação de diferentes séries escolares. Temas sobre Desenvolvimento 1999;7(42):37-42.

19. Liberman IY. Segmentation of the spoken word and reading acquisition. Bulletin of the Orton Society 1973,23:65-77.

20. Vellutino FR. Dyslexia: theory and research. Cambridge: MIT Press, 1979.

21. Vellutino FR. Dyslexia. Scientific Am 1987;256:20-27.

22. Lundberg I. Lack of phonological awareness: a critical factor in developmental dyslexia. In Von Euler C, Lundberg I, Lennerstrand G (eds). Brain and reading. London: Macmillan, 1989.

23. Snowling MJ, Hulme C. Developmental dyslexia and language disorders. In Blanken G, Dittmann J, Grimm H, Marshall C, Wallesch $\mathrm{CH}$ (eds). Linguistic disorders and pathologies: an international handbook. Berlin: de Gruyter, 1993:724-743.

24. Liberman IY. Segmentation of the spoken word and reading acquisition. Bull Orton Society 1973;23:65-77.

25. Gerber A. Problemas de aprendizagem relacionados à linguagem. Porto Alegre: Artes Médicas, 1996.

26. Humphreys P, Kaufmann, WE, Galaburda, AM. Developmental dyslexia in women: neuropathological findings in three cases. Ann Neurol 1990;28:727-738.

27. Hooper SR, Willis WG. Learning disability subtyping, neuropsychological foundations, conceptual models, and issues in clinical differention. New York: Springer-Verlag, 1989.

28. Mathias SC. Neuroimagem em neuropsicologia. In Nitrini R, Caramelli $\mathrm{P}$, Mansur LL (eds). Neuropsicologia: das bases anatômicas à reabilitação, São Paulo: FMUSP, 1996.

29. Howard D, Patterson K, Wise R, et al. The cortical localization of the lexicons. Brain 1992;115:1769-1782.

30. Galaburda AM, Sherman GF, Rosen GD, Aboitiz F, Geschwind N. Developmental dyslexia: four consecutive patients with cortical anomalies. Ann Neurol 1985;18:222-233. 